symptomatic relief of obstruction of the biliary tree. Although mortality following ERCP is high in patients with advanced age and a malignant diagnosis, a Cochrane review has shown that endoscopic stents have reduced complications and mortality compared with surgical bypass in inoperable pancreatic cancer. Furthermore metal stents have improved patency in biliary obstruction than plastic stents. Our aim was to evaluate patient outcomes following endoscopic metallic stenting at a specialist tertiary referral centre, including the need for re-intervention and mortality rates.

Methods We performed a retrospective audit and service evaluation for all endoscopic metallic biliary stent procedures at Queen's Medical Centre, Nottingham University Hospitals Trust over a 1year period during 2010 with patients receiving at least 1-year follow-up. Demographic data, the need for repeat intervention (either endoscopic or radiological), procedure-related complications and mortality were determined.

Results During 2010, 40/776 (5.2\%) patients undergoing ERCP had metallic biliary stents inserted; uncovered Zilver ${ }^{\circledR}$ stents (Wilson Cook, USA) $\mathrm{n}=38$ (95\%), covered Niti-S ${ }^{\circledR}$ stents (Taewoong Medical, S. Korea) $\mathrm{n}=2(5 \%)$. Of these $22(55 \%)$ were male and mean $( \pm \mathrm{SD})$ age was $73.1 \pm 12.3$ years. Final diagnosis was pancreatic cancer; $n=22$ (55\%), cholangiocarcinomas; $n=13$ (33\%), other malignancy; $\mathrm{n}=3(7 \%)$ and benign stricture; $\mathrm{n}=2(5 \%)$. Strictures were located either distally $n=25(63 \%)$, mid-duct strictures $n=4$ $(10 \%)$ or proximal/hilar strictures $n=11(27 \%)$. All patients underwent radiological imaging prior to ERCP. 22 patients (55\%) had undergone prior ERCP with the majority, 21/22 (95\%) patients, having confirmed cytological diagnosis of malignancy and 20/22 (91\%) patients having previous biliary stents in situ. These were predominantly plastic stents which had blocked or required stent exchange. All cause 1-year mortality was $80 \%$, with median (range) survival 120 (6-361) days. 7-day and 30-day mortality was 5\% and $13 \%$ respectively. There were no immediate reported complications at time of endoscopy. 9/40 (22.5\%) patients required further ERCP or percutaneous transhepatic cholangiography stenting procedures. All re-interventions were in patients with uncovered stents; 6 due to tumour in growth or stent occlusion and one due to stent misplacement.

Conclusion Although technical success and immediate complications were satisfactory, need for re-intervention was required in $22.5 \%$. Median survival and stent patency in this study is comparable to data from the Cochrane review, however further work is required to compare different metallic stent construction or other manoeuvres to reduce the need for re-intervention.

Competing interests None declared.

\section{PTU-237 COMPLICATIONS AND OUTCOMES FOLLOWING PERCUTANEOUS ENDOSCOPIC GASTROSTOMY (PEG) TUBE INSERTION: A SINGLE CENTRE EXPERIENCE}

doi:10.1136/gutjnl-2012-302514c.237

${ }^{1} \mathrm{~N}$ Magboul, ${ }^{*}{ }^{2} \mathrm{~A}$ Murugananthan, ${ }^{2} \mathrm{I}$ Kronborg. ${ }^{1}$ Department of Gastroenterology and Hepatology, Melbourne, Australia; ${ }^{2}$ Western Hospital, Melbourne, Australia

Introduction PEG insertion has a reported 30-day mortality of 19\%$24 \%{ }^{12}$ suggesting a significant number of inappropriate insertions that were unnecessary for good care. Despite this there are no consistent Australian guidelines regarding patient selection for this procedure. To try and identify patients who would not benefit from PEG insertion we conducted an audit of all PEG insertions undertaken at our hospital, with particular reference to indications, complications and outcome.

Methods PEG insertions from 15 January 2009 to 22 July 2011 were identified from an endoscopy database and then medical notes were reviewed. We assessed indication for PEG insertion, the use of prophylactic antibiotics, complications during the procedure, 30 day complications and longer term complication rates.

Results 28 patients were identified ( 13 male and 15 female). The mean age was 71.8 years. The most common indication for PEG insertion was dysphagia following a cerebrovascular accident (CVA) (65\%). Other indications included head and neck tumours (14\%), other neurological disorders and poor oral intake due to severe depression (21\%). $64 \%$ of patients received prophylactic antibiotics during the procedure with cephazolin compared to the recommended $100 \%$. Despite this low rate, no patients were re-admitted with PEG site infections. 30-day mortality was $23 \%$. In patients with CVAs 30 -day mortality was $45 \%$ vs $0 \%$ in patients with other indications ( $p=0.038$; Fishers exact test). Eleven patients had episodes of aspiration within 30 days of PEG insertion-the majority of these were from the CVA group (72\%). The median time between CVA and PEG insertion was 25.5 days (range 10-53 days). Overall survival at 1 year was 38\%. There were three cases in which the PEG fell out: two of the three were due to the patient removing it.

Conclusion Our data shows a significantly higher 30-day mortality in CVA patients as well as a higher frequency of aspiration in this group. This is in keeping with other studies ${ }^{3}$ and re-iterates the fact that insertion of a PEG does not prevent the development of aspiration pneumonia, which is a common misconception. It would therefore be reasonable to suggest a minimum period of 30 days of observation plus NG feeding in such patients to allow for any change in clinical condition.

Competing interests None declared.

\section{REFERENCES}

1. Janes SE, Price SG, Khan S. Percutaneous endoscopic gastrostomy: 30-day mortality trends and risk factors. J Postgrad Med 2005;51:23-8.

2. Mitchell SL, Tetroe JM. Survival after percutaneous endoscopic gastrostomy placement in older persons. J Gerontol A Biol Sci Med Sci 2000;55:735-9.

3. Marik PE. Primary care: aspiration pneumonitis and aspiration pneumonia. $N$ Eng/ $J$ Med 2001;344:665-71.

\section{PTU-238 PREP, NO PREP OR MORE PREP? A PROSPECTIVE RANDOMISED STUDY COMPARING TWO BOWEL PREPARATION REGIMES WITH NO PREPARATION ON QUALITY OF CAPSULE ENDOSCOPY}

doi:10.1136/gutjnl-2012-302514c.238

${ }^{1} \mathrm{~N}$ Magboul, ${ }^{*} \mathrm{~A}$ Murugananthan, ${ }^{2} \mathrm{~T}$ Hong, ${ }^{2} \mathrm{~J}$ French, ${ }^{2} \mathrm{R}$ Chen. ${ }^{1}$ Department of Gastroenterology and Hepatology, Melbourne, Australia; ${ }^{2}$ Western Hospital, Melbourne, Australia

Introduction Capsule endoscopy (CE) is a widely used method for evaluation of the small bowel. However it does have limitations; visualisation of the small bowel mucosa is often impaired due to the presence of food residue, air bubbles and bile pigments. ${ }^{1}$ The effect of bowel preparation on improving visualisation of the small bowel varies $^{2}$ and is inconvenient for patients. ${ }^{3}$ We aimed to prospectively evaluate the effects of two different bowel preparations on visualisation of the small bowel and on overall diagnostic yield compared with standard dietary changes.

Methods 51 patients (26 male/25 female; mean age 60.7 years) were randomised into three groups using the sealed envelope technique. Indications for CE were iron deficiency anaemia, obscure GI bleeding (occult and overt) and anaemia. Group $1(n=19)$ : Clear fluid day before procedure. Overnight fast. Group $2(n=12)$ : Clear fluid day before procedure. 2L PEG in afternoon of day prior to procedure. Overnight fast. Group $3(n=20)$ : Clear fluid day before procedure. 1L PEG and 1 sachet Picoprep in afternoon of day prior to procedure Overnight fast. CE were viewed by a single blinded examiner and 
adequacy of bowel preparation according to three categories (>80\% visualisation; $50 \%-80 \%$ visualisation).

Results

\begin{tabular}{lllllll}
\hline & $\begin{array}{l}\text { Mean } \\
\text { age }\end{array}$ & $\begin{array}{l}\text { Completion } \\
\text { rate (\%) }\end{array}$ & $\begin{array}{l}\text { Yield } \\
(\%)\end{array}$ & $\begin{array}{l}\text { Good SB } \\
\text { views (\%) }\end{array}$ & $\begin{array}{l}\text { Mean GTT } \pm \\
\text { SEM (min) }\end{array}$ & $\begin{array}{l}\text { Mean SBTT } \pm \\
\text { SEM (min) }\end{array}$ \\
\hline Gp 1 & 63.6 & 79 & 42.1 & 100 & $35.9 \pm 11.19$ & $254.8 \pm 24.83$ \\
Gp 2 & 57 & 83.3 & 41.6 & 81.2 & $87.5 \pm 47.79$ & $239.3 \pm 45.7$ \\
Gp 3 & 60 & 90 & 35 & 79 & $74.8 \pm 27.06$ & $211.5 \pm 24.14$ \\
p Value & & NS & NS & & NS & NS \\
\hline
\end{tabular}

Conclusion Our findings are in keeping with a recent meta-analysis which has shown no difference in CE completion rates, GTT and SBTT with purgative preparation. ${ }^{4}$ Our study shows a trend towards better caecal completion rates with bowel preparation involving PEG and Picoprep, but these results did not reach statistical significance. Overall diagnostic yield was similar in all three groups. Liquid diet, in combination with fasting, prior to CE is generally better tolerated by patients ${ }^{3}$ and our findings would support this as adequate preparation for $\mathrm{CE}$.

Competing interests None declared.

\section{REFERENCES}

1. Park SC, Keum B, Seo YS, et al. Effect of bowel preparation with polyethylene glycol on quality of capsule endoscopy. Dig Dis Sci 2011;56:1769-75.

2. Dai $\mathbf{N}$, Gubler $\mathrm{C}$, Hengstler $\mathrm{P}$, et al. Improved capsule endoscopy after bowel preparation. Gastrointest Endosc 2005;61:28-35.

3. Pons Beltrán V, González Suárez B, González Asanza C, et al. Evaluation of different bowel preparations for small bowel capsule endoscopy: a prospective, randomised, controlled study. Dig Dis Sci 2011;56:2900-5.

4. Rokkas T, Papaxoinis K, Triantafyllou K, et al. Does purgative preparation influence the diagnostic yield of small bowel video capsule endoscopy? : a meta-analysis. Am J Gastroenterol 2009;104:219-27.

\section{PTU-239 EUS ASSESSMENT OF LESIONS OF THE AMPULLA OF VATER: OF PARTICULAR VALUE IN LOW GRADE DYSPLASIA}

doi:10.1136/gutjnl-2012-302514c.239

K Roberts, N McCulloch, ${ }^{*}$ D Mayer, J Isaac, P Muiesan, S Bramhall, D Mirza, R Sutcliffe, C Forde, R Marudanayagam, B Mahon. Queen Elizabeth Hospital Birmingham, Birmingham, UK

Introduction Lesions of the ampulla of Vater are difficult to stage using conventional cross sectional imaging and endoscopy. An accurate diagnosis is essential as this permits endoscopic resection in dysplastic lesions preserving pancreatoduodenectomy for malignant cases. Endoscopic ultrasound has greater sensitivity and specificity than conventional imaging in staging lesions. To date its role in staging dysplastic lesions is unclear.

Methods Patients with adenomas or adenocarcinomas of the ampulla were identified from departmental databases over a 5-year period. Methods of presentation, investigation, treatment and outcome were recorded. Patients with no EUS were compared to those with EUS.
Results Of 58 patients, 27 were investigated with an EUS. There was no difference in age, sex or method of presentation between groups. The preoperative diagnosis was correct in $94 \%$ of cases in the EUS group vs $61 \%$ in the no EUS group ( $p=0.006)$. The sensitivity, specificity, positive and negative predictive values in the EUS group to correctly identify malignant lesions was 93, 100, 100 and $93 \%$ respectively. For the non-EUS group these values were 77,91 , 93 and $72 \%$. Every diagnosis of low grade dysplasia (LGD) was correct in the EUS group while these accounted for the majority of errors in the no EUS group. High grade dysplasia (HGD) was frequently understated.

Conclusion When added to existing investigations, EUS increases the accuracy of preoperative staging of ampullary lesions being particularly useful in cases of LGD. This permits safe endoscopic management of these cases. Cases of HGD must be reviewed carefully and considered for pancreatoduodenectomy.

Competing interests None declared.

\section{PTU-240 IMPROVING OUTCOMES OF ENDOSCOPIC RETROGRADE CHOLANGIO-PANCREATOGRAPHY WITH THE USE OF NEW TECHNIQUES IN A DISTRICT GENERAL HOSPITAL SETTING}

doi:10.1136/gutjnl-2012-302514c.240

N Owen, ${ }^{*}$ B Macfarlane, A King, A Leahy. Department of Gastroenterology, West Hertfordshire Hospitals NHS Trust, Watford, UK

Introduction Outcomes of endoscopic retrograde cholangio-pancreatography (ERCP) performed at West Hertfordshire Hospital NHS Trust (WHHT) in 2007 and 2011 were compared. The 2011 data followed introduction of the techniques of wire-guided cannulation and balloon sphincteroplasty. Outcomes measured included successful biliary cannulation on first ever ERCP, the use of precut, successful stenting of strictures, stone extraction and post ERCP pancreatitis rates. The number of referrals to a tertiary centre due to failed ERCP in both years was also calculated. The outcomes were compared with national data from the BSG ERCP audit of $2007 .{ }^{1}$

Methods Local data were obtained from all ERCP performed at WHHT in the years 2007 and 2011. Success of cannulation, precut use, successful stenting and stone extraction was collected from electronic ERCP reports. Post ERCP pancreatitis rates were obtained by confirming hyperamylasaemia post ERCP of at least three times the upper limit of normal requiring admission to hospital or prolongation of planned admission of $\geq 2$ nights, as per the BSG 2007 Audit definitions. ${ }^{1}$ Similar national outcomes were taken from the BSG 2007 Audit. $^{1}$

Results Results are summarised below:

A Fisher's exact test was performed to compare data. A statistically significant difference was found between the 2007 and 2011 groups ( $p$ value $<0.01$ ) when comparing successful stone extraction. Tertiary centre referrals due to failed ERCP fell from 12 in 2007 to 4 in 2011.

Conclusion The introduction of wire-guided cannulation has maintained high success rates of biliary cannulation with a reduction in

\begin{tabular}{|c|c|c|c|c|c|c|}
\hline ERCP data & $\begin{array}{l}\text { Successful biliary } \\
\text { cannulation on } \\
1 \text { st ever ERCP }\end{array}$ & Pre-cut use & Success of pre-cut & $\begin{array}{l}\text { Successful stenting of } \\
\text { stricture }\end{array}$ & $\begin{array}{l}\text { Successful stone } \\
\text { extraction }\end{array}$ & $\begin{array}{l}\text { Post ERCP } \\
\text { pancreatitis }\end{array}$ \\
\hline UK 2007 & 2684 of $3210(84 \%)$ & 465 of $5264(8.8 \%)$ & 303 of $465(65 \%)$ & 1341 of $1827(73 \%)$ & 1318 of $2114(62 \%)$ & 79 of $5264(1.5 \%)$ \\
\hline WHHT 2007 & 172 of $190(91 \%)$ & 12 of $218(5.5 \%)$ & 6 of $12(50 \%)$ & 46 of $54 \%(85 \%)$ & 61 of $91(67 \%)$ & 8 of $218(3.7 \%)$ \\
\hline WHHT 2011 & 180 of $193(93 \%)$ & 15 of $257(6 \%)$ & 11 of $15(73 \%)$ & 67 of $77(87 \%)$ & 97 of $116(84 \%)^{*}$ & 3 of $257(1.3 \%)$ \\
\hline
\end{tabular}

*Balloon sphincteroplasty used in 24 of $116(21 \%)$. 